\title{
Magic Graphoidal on Class of Trees
}

\author{
A. Nellai Murugan \\ Department of Mathematics, V.O.Chidambaram College, Tuticorin, Tamilnadu.
}

Keywords: Graphoidal Cover, Magic Graphoidal, Graphoidal Constant.

Abstract: B.D.Acharya and E. Sampathkumar [1] defined Graphoidal cover as partition of edge set of a graph $\mathrm{G}$ into internally disjoint paths (not necessarily open). The minimum cardinality of such cover is known as graphoidal covering number of $\mathrm{G}$.

Let $\mathrm{G}=\{\mathrm{V}, \mathrm{E}\}$ be a graph and let $\psi$ be a graphoidal cover of $\mathrm{G}$. Define $\mathrm{f}$ :

$\mathrm{V} \cup \mathrm{E} \rightarrow\{1,2, \ldots, \mathrm{p}+\mathrm{q}\}$ such that for every path $\mathrm{P}=(\mathrm{v} 0, \mathrm{v} 1, \mathrm{v} 2, \ldots \mathrm{vn})$ in $\psi$ with $\mathrm{f}^{*}(\mathrm{P})=$ $\mathrm{f}\left(\mathrm{v}_{0}\right)+\mathrm{f}\left(\mathrm{v}_{\mathrm{n}}\right)+\sum_{1}^{n} f(\mathrm{v} 1 \mathrm{i}-1 \mathrm{v})=\mathrm{k}$, a constant, where $\mathrm{f}^{*}$ is the induced labeling on $\psi$. Then, we say that $\mathrm{G}$ admits $\psi$ - magic graphoidal total labeling of $\mathrm{G}$.

A graph $\mathrm{G}$ is called magic graphoidal if there exists a minimum graphoidal cover $\psi$ of $\mathrm{G}$ such that $\mathrm{G}$ admits $\psi$ - magic graphoidal total labeling.

In this paper, we proved that $\left[\mathrm{P}_{\mathrm{n}} ; \mathrm{S}_{1}\right],\left[\mathrm{P}_{\mathrm{n}} ; \mathrm{S}_{2}\right], \mathrm{T}(\mathrm{n}), \mathrm{P}_{\mathrm{m}} \bigodot \mathrm{K}_{1,3}, \mathrm{P}_{\mathrm{m}} \bigodot 2 \mathrm{~K}_{1}$ and $\mathrm{K} 1, \mathrm{n}$ $2 \odot 1$ are magic graphoidal

\section{INTRODUCTION}

By a graph, we mean a finite simple and undirected graph. The vertex set and edge set of a graph $\mathrm{G}$ are denoted by $\mathrm{V}(\mathrm{G})$ and $\mathrm{E}(\mathrm{G})$ respectively. Terms and notations not used here are as in [3].

1.1. Definition : Let $S_{1}=\left(v_{0}, v_{1}\right)$ be a star and let $\left[P_{n} ; S_{1}\right]$ be the graph obtained from $n$ copies of $S_{1}$ and the path $P_{n}=\left(u_{1}, u_{2}, \ldots, u_{n}\right)$ by joining $u_{j}$ with the vertex $v_{0}$ of the $j^{\text {th }}$ copy of $S_{1}$ by means of an edge, for $1 \leq \mathrm{j} \leq \mathrm{n}$.

1.2. Definition : Let $S_{2}=\left(v_{0}, v_{1}, v_{2}\right)$ be a star and let $\left[P_{n} ; S_{2}\right]$ be the graph obtained from $n$ copies of $S_{2}$ and the path $P_{n}=\left(u_{1}, u_{2}, \ldots, u_{n}\right)$ by joining $u_{j}$ with the vertex $v_{0}$ of the $j^{\text {th }}$ copy of $S_{2}$ by means of an edge, for $1 \leq \mathrm{j} \leq \mathrm{n}$.

1.3. Defintion : Let $\mathrm{T}$ be any Tree. Denote the tree, obtained from $\mathrm{T}$ by considering two copies of $\mathrm{T}$ by adding an edge between them, by $\mathrm{T}_{(2)}$ and in general the graph obtained from $\mathrm{T}_{(\mathrm{n}-}$ 1) and $T$ by adding an edge between them is denoted by $T_{(n)}$.

1.4. Result [11] : For a Tree $T, \gamma(T)=n-1$ where $n$ is the number of pendent vertices of $G$.

\section{PRELIMINARIES}

Let $\mathrm{G}=\{\mathrm{V}, \mathrm{E}\}$ be a graph with $\mathrm{p}$ vertices and $\mathrm{q}$ edges. A graphoidal cover $\psi$ of $\mathrm{G}$ is a collection of (open) paths such that

(i) every edge is in exactly one path of $\psi$

(ii) every vertex is an interval vertex of atmost one path in $\psi$.

We define $\gamma(\mathrm{G})=\psi \in \zeta$ $\min _{\psi \in \zeta}|\psi|$,

where $\zeta$ is the collec tion of graphoidal covers $\psi$ of $\mathrm{G}$ and $\gamma$ is graphoidal covering number of $\mathrm{G}$. 
Let $\psi$ be a graphoidal cover of $\mathrm{G}$. Then we say that $\mathrm{G}$ admits $\psi$ - magic graphoidal total labeling of $\mathrm{G}$ if there exists a bijection $\mathrm{f}: \mathrm{V} \cup \mathrm{E} \rightarrow\{1,2, \ldots, \mathrm{p}+\mathrm{q}\}$ such that for every path $\mathrm{P}$ $=\left(\mathrm{v}_{0} \mathrm{v}_{1} \mathrm{v}_{2} \ldots \mathrm{v}_{\mathrm{n}}\right)$ in $\psi$, then, $\mathrm{f}^{*}(\mathrm{P})=\mathrm{f}\left(\mathrm{v}_{0}\right)+\mathrm{f}\left(\mathrm{v}_{\mathrm{n}}\right)+\sum_{1}^{n} f\left(\mathrm{v}_{1 \mathrm{i}-1 \mathrm{~V}}\right)=\mathrm{k}$, a constant, where $\mathrm{f}^{*}$ is the induced labeling of $\psi$. A graph $\mathrm{G}$ is called magic graphoidal if there exists a minimum graphoidal cover $\psi$ of $\mathrm{G}$ such that $\mathrm{G}$ admits $\psi$ - magic graphoidal total labeling.

\section{Magical Graphoidal on Trees}

3.1. Theorem : $\left[\mathrm{P}_{\mathrm{n}} ; \mathrm{S}_{1}\right]$, (n - even) is magic graphoidal.

Proof: Let $G=\left[P_{n} ; S_{1}\right]$

Let $\mathrm{V}(\mathrm{G})=\left\{\mathrm{u}_{\mathrm{i}}, \mathrm{v}_{\mathrm{i}}, \mathrm{w}_{\mathrm{i}}: 1 \leq \mathrm{i} \leq \mathrm{n}\right\}$ and

$$
\mathrm{E}(\mathrm{G})=\left\{\left[\left(\mathrm{u}_{\mathrm{i}} \mathrm{v}_{\mathrm{i}}\right) \cup\left(\mathrm{v}_{\mathrm{i}} \mathrm{w}_{\mathrm{i}}\right): 1 \leq \mathrm{i} \leq \mathrm{n}\right] \cup\left[\left(\mathrm{u}_{\mathrm{i}} \mathrm{u}_{\mathrm{i}+1}\right): 1 \leq \mathrm{i} \leq \mathrm{n}-1\right]\right\}
$$

Define $\mathrm{f}: \mathrm{V} \cup \mathrm{E} \rightarrow\{1,2, \ldots, \mathrm{p}+\mathrm{q}\}$ by

$$
\begin{aligned}
& \mathrm{f}\left(\mathrm{w}_{1}\right) \quad=1 \\
& \mathrm{f}\left(\mathrm{w}_{1} \mathrm{v}_{1}\right) \quad=2 \\
& \mathrm{f}\left(\mathrm{v}_{1} \mathrm{u}_{1}\right) \quad=3 \\
& \mathrm{f}\left(\mathrm{u}_{\mathrm{i}+1}\right) \quad=6+\mathrm{i} \quad 1 \leq \mathrm{i} \leq \mathrm{n}-2 \\
& \mathrm{f}\left(\mathrm{w}_{\mathrm{i}+1}\right)=6 \mathrm{n}-\mathrm{i} \quad 1 \leq \mathrm{i} \leq \mathrm{n}-1 \\
& \mathrm{f}\left(\mathrm{v}_{\mathrm{i}+1} \mathrm{w}_{\mathrm{i}+1}\right)=4 \mathrm{n}+1+\mathrm{i} \quad 1 \leq \mathrm{i} \leq \mathrm{n}-1 \\
& \mathrm{f}\left(\mathrm{u}_{\mathrm{i}+1} \mathrm{v}_{\mathrm{i}+1}\right)=3 \mathrm{n}+3-2 \mathrm{i} \quad 1 \leq \mathrm{i} \leq(\mathrm{n} \backslash 2)-1 \\
& \mathrm{f}\left(\mathrm{u}_{\frac{\mathrm{n}}{2}+\mathrm{i}} \mathrm{v}_{\frac{\mathrm{n}}{2}+\mathrm{i}}\right)=3 \mathrm{n}+4-2 \mathrm{i} \quad 1 \leq \mathrm{i} \leq \frac{\mathrm{n}}{2} \\
& \mathrm{f}\left(\mathrm{u}_{\mathrm{i}} \mathrm{u}_{\mathrm{i}+1}\right) \quad=\frac{3 \mathrm{n}}{2}+4+\mathrm{i} \quad 1 \leq \mathrm{i} \leq \frac{\mathrm{n}}{2}-1 \\
& \mathrm{f}\left(\mathrm{u}_{\frac{\mathrm{n}}{2}-1+\mathrm{i}} \mathrm{u}_{\frac{\mathrm{n}}{2}+\mathrm{i}}\right)=\mathrm{n}+4+\mathrm{i} \quad 1 \leq \mathrm{i} \leq \frac{\mathrm{n}}{2}
\end{aligned}
$$

Let $\psi=\left\{\mathrm{P}_{1}=\left(\mathrm{w}_{1}, \mathrm{v}_{1}, \mathrm{u}_{1}, \mathrm{u}_{2}, \mathrm{v}_{2}, \mathrm{w}_{2}\right), \mathrm{P}_{2}=\left(\mathrm{u}_{\mathrm{i}}, \mathrm{u}_{\mathrm{i}+1}, \mathrm{v}_{\mathrm{i}+1}, \mathrm{w}_{\mathrm{i}+1}\right): 2 \leq \mathrm{i} \leq \mathrm{n}-1\right\}$$$
\mathrm{f}^{*}\left[\mathrm{P}_{1}\right]=\mathrm{f}\left(\mathrm{w}_{1}\right)+\mathrm{f}\left(\mathrm{w}_{2}\right)+\mathrm{f}\left(\mathrm{w}_{1} \mathrm{v}_{1}\right)+\mathrm{f}\left(\mathrm{v}_{1} \mathrm{u}_{1}\right)+\mathrm{f}\left(\mathrm{u}_{1} \mathrm{u}_{2}\right)+\mathrm{f}\left(\mathrm{u}_{2} \mathrm{v}_{2}\right)+\mathrm{f}\left(\mathrm{v}_{2} \mathrm{w}_{2}\right)
$$$$
=1+6 n-1+2+3+\frac{3 n}{2}+4+1+3 n+3-2+4 n+2
$$$$
=13 n+\frac{3 n}{2}+13
$$

For $2 \leq \mathrm{i} \leq(\mathrm{n} \backslash 2)-1$,

$$
\begin{aligned}
f^{*}\left[P_{2}\right]= & f\left(u_{i}\right)+f\left(w_{i+1}\right)+f\left(u_{i} u_{i+1}\right)+f\left(u_{i+1} v_{i+1}\right)+f\left(v_{i+1} w_{i+1}\right) \\
& =6+i-1+6 n-i+(3 n \backslash 2)+4+i+3 n+3-2 i+4 n+1+i \\
& =13 n+(3 n \backslash 2)+13-----(B)
\end{aligned}
$$

For $(\mathrm{n} \backslash 2) \leq \mathrm{i} \leq \mathrm{n}-1$, 
$f^{*}\left[P_{2}\right]=f\left(u_{i}\right)+f\left(w_{i+1}\right)+f\left(u_{i} u_{i+1}\right)+f\left(u_{i+1} v_{i+1}\right)+f\left(v_{i+1} w_{i+1}\right)$

$=6+i-1+6 n-i+n+4+i-(n \backslash 2)+1+3 n+4-2(i+1-(n \backslash 2))+4 n+1+i$

$=13 \mathrm{n}+(3 \mathrm{n} \backslash 2)+13$

From (A), (B) and (C), we conclude that $\mathrm{G}$ admits $\psi$ - magic graphoidal total labeling. Hence, $\left[\mathrm{P}_{\mathrm{n}}\right.$ $\left.; \mathrm{S}_{1}\right]$, (n - even) is magic graphoidal.

For example, consider the graph $\left[\mathrm{P}_{6} ; \mathrm{S}_{1}\right]$ shown in figure 1.

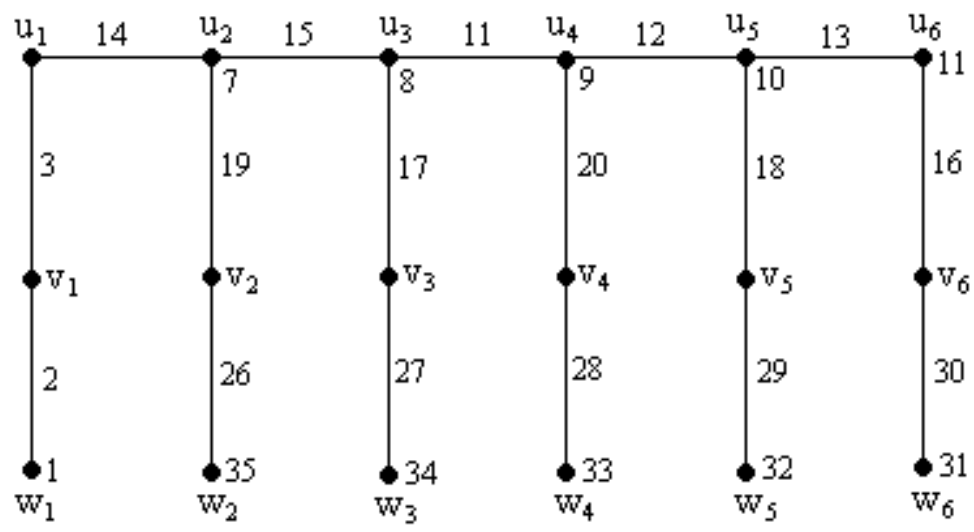

Figure $1[\mathrm{P} 6 ; \mathrm{S} 1]$

Clearly, $\psi=\left\{\left(\mathrm{w}_{1}, \mathrm{v}_{1}, \mathrm{u}_{1}, \mathrm{u}_{2}, \mathrm{v}_{2}, \mathrm{w}_{2}\right),\left(\mathrm{u}_{2}, \mathrm{u}_{3}, \mathrm{v}_{3}, \mathrm{w}_{3}\right),\left(\mathrm{u}_{3}, \mathrm{u}_{4}, \mathrm{v}_{4}, \mathrm{w}_{4}\right), \quad\left(\mathrm{u}_{4}, \mathrm{u}_{5}, \mathrm{v}_{5}, \mathrm{w}_{5}\right),\left(\mathrm{u}_{5}, \mathrm{u}_{6}, \mathrm{v}_{6}, \mathrm{w}_{6}\right)\right\}$ is a minimum graphoidal cover and $\left[\mathrm{P}_{6} ; \mathrm{S}_{1}\right]$ is magic graphoidal. Here the constant $\mathrm{K}=100$

3.2. Theorem : $\left[\mathrm{P}_{\mathrm{n}} ; \mathrm{S}_{1}\right],(\mathrm{n}-\mathrm{odd})$ is magic graphoidal.

Proof: Let $\mathrm{G}=\left[\mathrm{P}_{\mathrm{n}} ; \mathrm{S}_{1}\right]$

Let $\mathrm{V}(\mathrm{G})=\left\{\mathrm{u}_{\mathrm{i}}, \mathrm{v}_{\mathrm{i}}, \mathrm{w}_{\mathrm{i}}: \quad 1 \leq \mathrm{i} \leq \mathrm{n}\right\}$ and

$$
\mathrm{E}(\mathrm{G})=\left\{\left[\left(\mathrm{u}_{\mathrm{i}} \mathrm{v}_{\mathrm{i}}\right) \cup\left(\mathrm{v}_{\mathrm{i}} \mathrm{W}_{\mathrm{i}}\right): 1 \leq \mathrm{i} \leq \mathrm{n}\right] \cup\left[\left(\mathrm{u}_{\mathrm{i}} \mathrm{u}_{\mathrm{i}+1}\right): 1 \leq \mathrm{i} \leq \mathrm{n}-1\right]\right\}
$$

Define $\mathrm{f}: \mathrm{V} \cup \mathrm{E} \rightarrow\{1,2, \ldots, \mathrm{p}+\mathrm{q}\}$ by

$$
\begin{array}{llc}
\mathrm{f}\left(\mathrm{w}_{1}\right) & =1 & \\
\mathrm{f}\left(\mathrm{w}_{1} \mathrm{v}_{1}\right) & =2 & \\
\mathrm{f}\left(\mathrm{v}_{1} \mathrm{u}_{1}\right) & =3 & 1 \leq \mathrm{i} \leq(\mathrm{n}-1) \backslash 2 \\
\mathrm{f}\left(\mathrm{u}_{\mathrm{i}+1}\right) & =5+2 \mathrm{i} & 1 \leq \mathrm{i} \leq \mathrm{n}-1 \\
\mathrm{f}\left(\mathrm{w}_{\mathrm{i}+1}\right) & =6 \mathrm{n}-\mathrm{i} & 1 \leq \mathrm{i} \leq \mathrm{n}-1 \\
\mathrm{f}\left(\mathrm{v}_{\mathrm{i}+1} \mathrm{w}_{\mathrm{i}+1}\right) & =4 \mathrm{n}+1+\mathrm{i} & \\
\mathrm{f}\left(\mathrm{u}_{1} \mathrm{u}_{2}\right) & =\mathrm{n}+5 & \\
\mathrm{f}\left(\mathrm{u}_{\mathrm{i}+1} \mathrm{u}_{\mathrm{i}+2}\right) & =\frac{3 \mathrm{n}+11}{2}-\mathrm{i} & 1 \leq \mathrm{i} \leq \frac{\mathrm{n}-1}{2} \\
\mathrm{f}\left(\mathrm{u}_{\mathrm{n}+1-\mathrm{i}} \mathrm{u}_{\mathrm{n}-\mathrm{i}}\right) & =\frac{3 \mathrm{n}+9}{2}+\mathrm{i} & 1 \leq \mathrm{i} \leq \frac{\mathrm{n}-3}{2} \\
\mathrm{f}\left(\mathrm{u}_{\mathrm{i}+2} \mathrm{v}_{\mathrm{i}+2}\right) & =3 \mathrm{n}+2-\mathrm{i} & 1 \leq \mathrm{i} \leq \mathrm{n}-2 \\
\mathrm{f}\left(\mathrm{u}_{2} \mathrm{v}_{2}\right) & \frac{7 \mathrm{n}+3}{2} &
\end{array}
$$


Let $\psi=\left\{\mathrm{P}_{1}=\left(\mathrm{w}_{1}, \mathrm{v}_{1}, \mathrm{u}_{1}, \mathrm{u}_{2}, \mathrm{v}_{2}, \mathrm{w}_{2}\right), \mathrm{P}_{2}=\left(\mathrm{u}_{\mathrm{i}}, \mathrm{u}_{\mathrm{i}+1}, \mathrm{v}_{\mathrm{i}+1}, \mathrm{w}_{\mathrm{i}+1}\right): 2 \leq \mathrm{i} \leq \mathrm{n}-1\right\}$

$\mathrm{f}^{*}\left[\mathrm{P}_{1}\right]=\mathrm{f}\left(\mathrm{w}_{1}\right)+\mathrm{f}\left(\mathrm{w}_{2}\right)+\mathrm{f}\left(\mathrm{w}_{1} \mathrm{v}_{1}\right)+\mathrm{f}\left(\mathrm{v}_{1} \mathrm{u}_{1}\right)+\mathrm{f}\left(\mathrm{u}_{1} \mathrm{u}_{2}\right)+\mathrm{f}\left(\mathrm{u}_{2} \mathrm{v}_{2}\right)+\mathrm{f}\left(\mathrm{v}_{2} \mathrm{w}_{2}\right)$

$$
\begin{aligned}
& =1+6 n-1+2+3+n+5+\frac{7 n+3}{2}+4 n+1+1 \\
& =14 n+\frac{n+1}{2}+13-- \text { (A) }
\end{aligned}
$$

For $2 \leq \mathrm{i}<\frac{\mathrm{n}+1}{2}$

$$
\begin{aligned}
\mathrm{f}^{*}\left[\mathrm{P}_{2}\right] & =\mathrm{f}\left(\mathrm{u}_{\mathrm{i}}\right)^{2}+\mathrm{I}\left(\mathrm{w}_{\mathrm{i}+1}\right)+\mathrm{f}\left(\mathrm{u}_{\mathrm{i}} \mathrm{u}_{\mathrm{i}+1}\right)+\mathrm{f}\left(\mathrm{u}_{\mathrm{i}+1} \mathrm{v}_{\mathrm{i}+1}\right)+\mathrm{f}\left(\mathrm{v}_{\mathrm{i}+1} \mathrm{w}_{\mathrm{i}+1}\right) \\
& =5+2(\mathrm{i}-1)+6 \mathrm{n}-\mathrm{i}+\frac{3 \mathrm{n}+11}{2}-(\mathrm{i}-1)+3 \mathrm{n}+2-(\mathrm{i}-1)+4 \mathrm{n}+1+\mathrm{i} \\
& =14 \mathrm{n}+\frac{\mathrm{n}+1}{2}+13-\text { (B) }
\end{aligned}
$$

For $\frac{\mathrm{n}+1}{2} \leq \mathrm{i} \leq \mathrm{n}-1$,

$$
\begin{aligned}
f^{*}\left[P_{2}\right] & =f\left(u_{i}\right)+f\left(w_{i+1}\right)+f\left(u_{i} u_{i+1}\right)+f\left(u_{i+1} v_{i+1}\right)+f\left(v_{i+1} w_{i+1}\right) \\
& =6+2\left(i-\frac{n+1}{2}\right)+6 n-i+\frac{3 n+9}{2}+(n-i)+3 n+2-(i-1)+4 n+1+i \\
& =14 n+\frac{n+1}{2}+13-(C)
\end{aligned}
$$

From (A), (B) and (C), we conclude that $\psi$ is minimum magic graphoidal cover Hence, $\left[\mathrm{P}_{\mathrm{n}} ; \mathrm{S}_{1}\right]$, ( $\mathrm{n}$ - even) is magic graphoidal.

For example, consider the graph $\left[\mathrm{P}_{7} ; \mathrm{S}_{1}\right]$ shown in figure 2 .

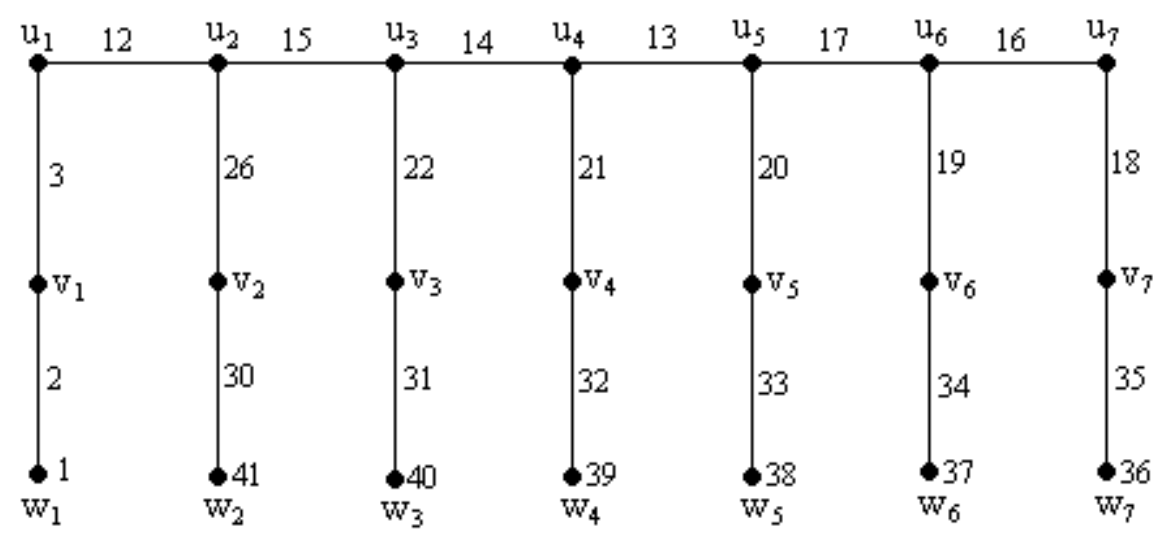

Figure $2\left[\mathrm{P}_{7} ; \mathrm{S}_{1}\right]$

Clearly, $\psi=\left\{\left(\mathrm{w}_{1}, \mathrm{v}_{1}, \mathrm{u}_{1}, \mathrm{u}_{2}, \mathrm{v}_{2}, \mathrm{w}_{2}\right), \quad\left(\mathrm{u}_{2}, \mathrm{u}_{3}, \mathrm{v}_{3}, \mathrm{w}_{3}\right), \quad\left(\mathrm{u}_{3}, \mathrm{u}_{4}, \mathrm{v}_{4}, \mathrm{w}_{4}\right), \quad\left(\mathrm{u}_{4}, \mathrm{u}_{5}, \mathrm{v}_{5}, \mathrm{w}_{5}\right), \quad\left(\mathrm{u}_{5}, \mathrm{u}_{6}, \mathrm{v}_{6}, \mathrm{w}_{6}\right)\right.$, $\left.\left(\mathrm{u}_{6}, \mathrm{u}_{7}, \mathrm{v}_{7}, \mathrm{w}_{7}\right)\right\}$ is a minimum graphoidal cover and $\left[\mathrm{P}_{7} ; \mathrm{S}_{1}\right]$ is magic graphoidal. Here the constant $\mathrm{K}=115$.

3.3. Theorem : $\left[\mathrm{P}_{\mathrm{n}} ; \mathrm{S}_{2}\right]$ is magic graphoidal.

Proof: Let $\mathrm{G}=\left[\mathrm{P}_{\mathrm{n}} ; \mathrm{S}_{2}\right]$

$$
\mathrm{V}(\mathrm{G})=\left\{\left(\mathrm{u}_{\mathrm{i}}, \mathrm{v}_{\mathrm{i}}\right): 1 \leq \mathrm{i} \leq \mathrm{n}\right\}
$$




$$
\begin{array}{r}
\mathrm{E}(\mathrm{G})=\left\{\left[\left(\mathrm{u}_{\mathrm{i}} \mathrm{u}_{\mathrm{i}+1}\right): 1 \leq \mathrm{i} \leq \mathrm{n}-1\right] \cup\left[\left(\mathrm{u}_{\mathrm{i}} \mathrm{v}_{\mathrm{i}}\right): 1 \leq \mathrm{i} \leq \mathrm{n}\right]\right. \\
\left.\cup\left[\left(\mathrm{v}_{\mathrm{i}} \mathrm{v}_{\mathrm{i} 1}\right) \cup\left(\mathrm{v}_{\mathrm{i}} \mathrm{v}_{\mathrm{i} 2}\right): 1 \leq \mathrm{i} \leq \mathrm{n}\right]\right\}
\end{array}
$$

Define $\mathrm{f}: \mathrm{V} \cup \mathrm{E} \rightarrow\{1,2, \ldots, \mathrm{p}+\mathrm{q}\}$ by

$$
\begin{array}{ll}
\mathrm{f}\left(\mathrm{v}_{1}\right) \quad=1 & \\
\mathrm{f}\left(\mathrm{u}_{1} \mathrm{v}_{1}\right) \quad=2 \mathrm{f} & \\
\left(\mathrm{u}_{1}\right) \quad=3 & 1 \leq \mathrm{i} \leq \mathrm{n}-2 \\
\mathrm{f}\left(\mathrm{u}_{\mathrm{i}+1}\right)=3+\mathrm{i} & \\
\mathrm{f}\left(\mathrm{u}_{\mathrm{n}}\right)=\mathrm{p}+\mathrm{q} & 1 \leq \mathrm{i} \leq \mathrm{n} \\
\mathrm{f}\left(\mathrm{v}_{\mathrm{i} 1}\right)=\mathrm{n}+1+\mathrm{i} & 1 \leq \mathrm{i} \leq \mathrm{n}-1 \\
\mathrm{f}\left(\mathrm{v}_{\mathrm{i}+1}\right)=8 \mathrm{n}-1-\mathrm{i} & 1 \leq \mathrm{i} \leq \mathrm{n} \\
\mathrm{f}\left(\mathrm{v}_{\mathrm{i} 2}\right)=7 \mathrm{n}-\mathrm{i} & 1 \leq \mathrm{i} \leq \mathrm{n} \\
\mathrm{f}\left(\mathrm{v}_{\mathrm{i}} \mathrm{v}_{\mathrm{i} 2}\right)=5 \mathrm{n}-1+\mathrm{i} & 1 \leq \mathrm{i} \leq \mathrm{n}-1 \\
\mathrm{f}\left(\mathrm{u}_{\mathrm{i}+1} \mathrm{v}_{\mathrm{i}+1}\right)=4 \mathrm{n}+\mathrm{i} & 1 \leq \mathrm{i} \leq \mathrm{n}-1 \\
\mathrm{f}\left(\mathrm{u}_{\mathrm{i}} \mathrm{u}_{\mathrm{i}+1}\right)=4 \mathrm{n}+1-\mathrm{i} & 1 \leq \mathrm{i} \leq \mathrm{n} \\
\mathrm{f}\left(\mathrm{v}_{\mathrm{i}} \mathrm{v}_{\mathrm{i} 1}\right)=3 \mathrm{n}+2-\mathrm{i} &
\end{array}
$$

Let $\psi=\left\{\mathrm{P}_{1}=\left[\left(\mathrm{v}_{\mathrm{i} 1}, \mathrm{v}_{\mathrm{i}}, \mathrm{v}_{\mathrm{i} 2}\right): 1 \leq \mathrm{i} \leq \mathrm{n}\right], \mathrm{P}_{2}=\left(\mathrm{v}_{1}, \mathrm{u}_{1}, \mathrm{u}_{2}, \mathrm{v}_{2}\right), \mathrm{P}_{3}=\left[\left(\mathrm{u}_{\mathrm{i}}, \mathrm{u}_{\mathrm{i}+1}, \mathrm{v}_{\mathrm{i}+1}\right): 2 \leq \mathrm{i} \leq \mathrm{n}-1\right]\right\}$

For $1 \leq \mathrm{i} \leq \mathrm{n}$,

$$
\begin{aligned}
\mathrm{f}^{*}\left[\mathrm{P}_{1}\right] & =\mathrm{f}\left(\mathrm{v}_{\mathrm{i} 1}\right)+\mathrm{f}\left(\mathrm{v}_{\mathrm{i} 2}\right)+\mathrm{f}\left(\mathrm{v}_{\mathrm{i} 1} \mathrm{v}_{\mathrm{i}}\right)+\mathrm{f}\left(\mathrm{v}_{\mathrm{i}} \mathrm{v}_{\mathrm{i} 2}\right) \\
& =\mathrm{n}+1+\mathrm{i}+7 \mathrm{n}-\mathrm{i}+3 \mathrm{n}+2-\mathrm{i}+5 \mathrm{n}-1+\mathrm{i} \\
& =16 \mathrm{n}+2-----(\mathbf{A}) \\
\mathrm{f}^{*}\left[\mathrm{P}_{2}\right] & =\mathrm{f}\left(\mathrm{v}_{1}\right)+\mathrm{f}\left(\mathrm{v}_{2}\right)+\mathrm{f}\left(\mathrm{v}_{1} \mathrm{u}_{1}\right)+\mathrm{f}\left(\mathrm{u}_{1} \mathrm{u}_{2}\right)+\mathrm{f}\left(\mathrm{u}_{2} \mathrm{v}_{2}\right) \\
& =1+8 \mathrm{n}-2+2+4 \mathrm{n}+4 \mathrm{n}+1 \\
& =16 \mathrm{n}+2-- \text { (B) }
\end{aligned}
$$

For $2 \leq \mathrm{i} \leq \mathrm{n}-1$,

$$
\begin{aligned}
f^{*}\left[P_{3}\right] & =f\left(u_{i}\right)+f\left(v_{i+1}\right)+f\left(u_{i} u_{i+1}\right)+f\left(u_{i+1} v_{i+1}\right) \\
& =3+i-1+8 n-1-i+4 n+1-i+4 n+i \\
& =16 n+2
\end{aligned}
$$

From (A), (B) and (C), we conclude that $\mathrm{G}$ admits $\psi$ - magic graphoidal total labeling. Hence, $\left[\mathrm{P}_{\mathrm{n}}\right.$ $\left.; \mathrm{S}_{2}\right]$ is magic graphoidal.

For example, consider the graphs $\left[\mathrm{P}_{3} ; \mathrm{S}_{2}\right]$ and $\left[\mathrm{P}_{4} ; \mathrm{S}_{2}\right]$ shown in figure 3.1 and 3.2.

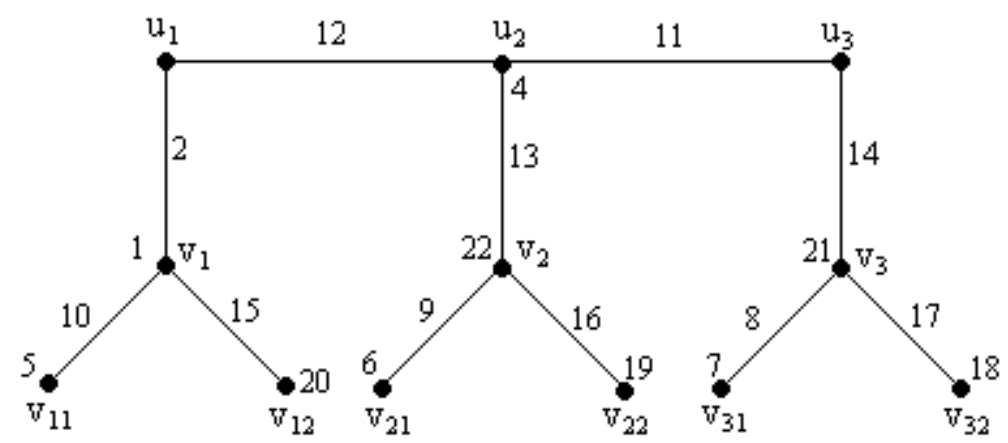

Figure 3.1 $\left[\mathrm{P}_{3} ; \mathrm{S}_{2}\right]$

Clearly, $\psi=\left\{\left(\mathrm{v}_{11}, \mathrm{v}_{1}, \mathrm{v}_{12}\right),\left(\mathrm{v}_{21}, \mathrm{v}_{2}, \mathrm{v}_{22}\right),\left(\mathrm{v}_{31}, \mathrm{v}_{3}, \mathrm{v}_{32}\right),\left(\mathrm{v}_{1}, \mathrm{u}_{1}, \mathrm{u}_{2}, \mathrm{v}_{2}\right), \quad\left(\mathrm{u}_{2}, \mathrm{u}_{3}, \mathrm{v}_{3}\right)\right\}$ is a minimum graphoidal cover and $\left[\mathrm{P}_{3} ; \mathrm{S}_{2}\right]$ is magic graphoidal. Here the constant $\mathrm{K}=50$ 


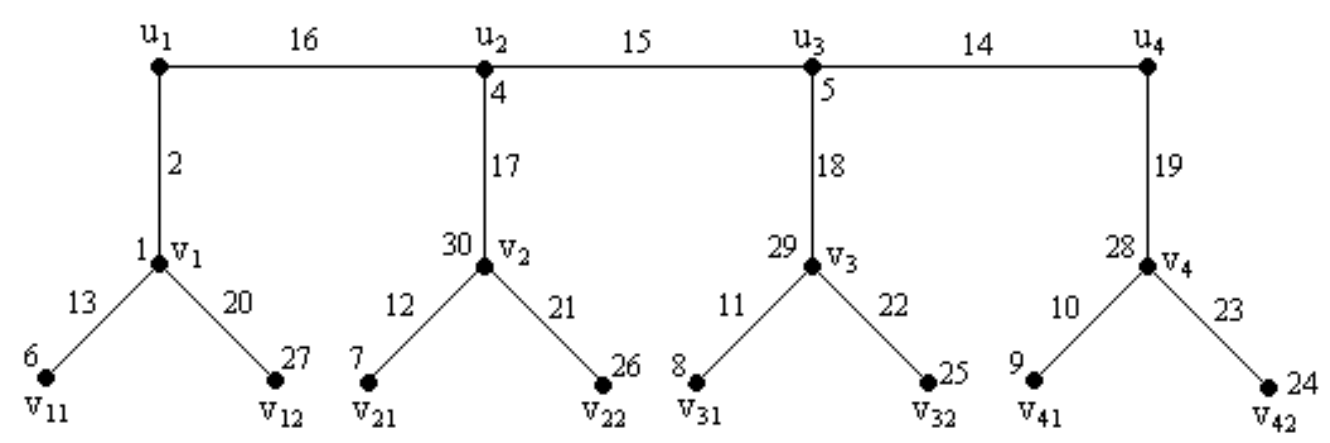

Figure 3.2 $\left[\mathrm{P}_{4} ; \mathrm{S}_{2}\right]$

Clearly, $\psi=\left\{\left(\mathrm{v}_{11}, \mathrm{v}_{1}, \mathrm{v}_{12}\right),\left(\mathrm{v}_{21}, \mathrm{v}_{2}, \mathrm{v}_{22}\right),\left(\mathrm{v}_{31}, \mathrm{v}_{3}, \mathrm{v}_{32}\right),\left(\mathrm{v}_{41}, \mathrm{v}_{4}, \mathrm{v}_{42}\right),\left(\mathrm{v}_{1}, \mathrm{u}_{1}, \mathrm{u}_{2}, \mathrm{v}_{2}\right),\left(\mathrm{u}_{2}, \mathrm{u}_{3}, \mathrm{v}_{3}\right),\left(\mathrm{u}_{3}, \mathrm{u}_{4}, \mathrm{v}_{4}\right)\right\}$ is a minimum graphoidal cover and $\left[\mathrm{P}_{4} ; \mathrm{S}_{2}\right]$ is magic graphoidal. Here the constant $\mathrm{K}=66$

3.4. Theorem : For a Tree, $\mathrm{T}_{(\mathrm{n})}$ is magic graphoidal.

\section{Proof :}

Let $\mathrm{T}_{(\mathrm{n})}$ be a graph such that

$\mathrm{V}\left[\mathrm{T}_{(\mathrm{n})}\right]=\left\{\mathrm{u}_{\mathrm{i} 1}, \mathrm{u}_{\mathrm{i} 2}, \mathrm{u}_{\mathrm{i} 3}, \mathrm{u}_{\mathrm{i} 4}, \mathrm{u}_{\mathrm{i} 5}: 1 \leq \mathrm{i} \leq \mathrm{n}\right\}$ and

$\left.\mathrm{E}\left[\mathrm{T}_{(\mathrm{n})}\right]=\left\{\left[\left(\mathrm{u}_{\mathrm{i} 1} \mathrm{u}_{\mathrm{i} 2}\right),\left(\mathrm{u}_{\mathrm{i} 2} \mathrm{u}_{\mathrm{i} 3}\right),\left(\mathrm{u}_{\mathrm{i} 3} \mathrm{u}_{\mathrm{i} 4}\right),\left(\mathrm{u}_{\mathrm{i} 4} \mathrm{u}_{\mathrm{i}}\right): 1 \leq \mathrm{i} \leq \mathrm{n}\right] \cup\left[\left(\mathrm{u}_{\mathrm{in}} \mathrm{u}_{\mathrm{i}+1 \mathrm{n}}\right): 1 \leq \mathrm{i} \leq \mathrm{n}-1\right)\right]\right\}$

Define $\mathrm{f}: \mathrm{V} \cup \mathrm{E} \rightarrow\{1,2, \ldots, \mathrm{p}+\mathrm{q}\}$ by

$\begin{array}{lll}\mathrm{f}\left(\mathrm{u}_{\mathrm{i} 1}\right) & =\mathrm{i} & 1 \leq \mathrm{i} \leq \mathrm{n} \\ \mathrm{f}\left(\mathrm{u}_{\mathrm{i} 3}\right) & =\mathrm{n}+1 & \\ \mathrm{f}\left(\mathrm{u}_{\mathrm{i} 3} \mathrm{u}_{\mathrm{i} 5}\right) & =\mathrm{n}+2 & \\ \mathrm{f}\left(\mathrm{u}_{\mathrm{i} 1} \mathrm{u}_{\mathrm{i} 2}\right) & =2 \mathrm{n}+3-\mathrm{i} & 1 \leq \mathrm{i} \leq \mathrm{n} \\ \mathrm{f}\left(\mathrm{u}_{\mathrm{i}+2,5} \mathrm{u}_{\mathrm{i}+3,5}\right) & =5 \mathrm{n}-3-2 \mathrm{i} & 1 \leq \mathrm{i} \leq \mathrm{n}-3 \\ \mathrm{f}\left(\mathrm{u}_{\mathrm{n}+1-\mathrm{i}, 5} \mathrm{u}_{\mathrm{n}-\mathrm{i}, 5}\right) & =4 \mathrm{n}-1+\mathrm{i} & \mathrm{n}-2 \leq \mathrm{i} \leq \mathrm{n}-1 \\ \mathrm{f}\left(\mathrm{u}_{\mathrm{n}+1-\mathrm{i}, 2} \mathrm{u}_{\mathrm{n}+1-\mathrm{i}, 3}\right) & =5 \mathrm{n}-2+\mathrm{i} & 1 \leq \mathrm{i} \leq \mathrm{n} \\ \mathrm{f}\left(\mathrm{u}_{\mathrm{n}+1-\mathrm{i}, 3} \mathrm{u}_{\mathrm{n}+1-\mathrm{i}, 4}\right) & =6 \mathrm{n}-2+\mathrm{i} & 1 \leq \mathrm{i} \leq \mathrm{n} \\ \mathrm{f}\left(\mathrm{u}_{\mathrm{n}+1-\mathrm{i}, 3} \mathrm{u}_{\mathrm{n}+1-\mathrm{i}, 5}\right) & =7 \mathrm{n}-2+\mathrm{i} & 1 \leq \mathrm{i} \leq \mathrm{n}-1 \\ \mathrm{f}\left(\mathrm{u}_{\mathrm{i} 4}\right) & =8 \mathrm{n}-2+2(\mathrm{i}-1) & 1 \leq \mathrm{i} \leq \mathrm{n} \\ \mathrm{f}\left(\mathrm{u}_{\mathrm{i}+1,3}\right) & =8 \mathrm{n}-1+2(\mathrm{i}-1) & 1 \leq \mathrm{i} \leq \mathrm{n}-1\end{array}$

Let $\psi=\left\{\mathrm{P}_{1}=\left(\mathrm{u}_{\mathrm{i} 1}, \mathrm{u}_{\mathrm{i} 2}, \mathrm{u}_{\mathrm{i} 3}, \mathrm{u}_{\mathrm{i} 4}\right), \mathrm{P}_{2}=\left(\mathrm{u}_{\mathrm{i} 3}, \mathrm{u}_{\mathrm{i} 5}, \mathrm{u}_{25}, \mathrm{u}_{23}\right), \mathrm{P}_{3}=\left[\left(\mathrm{u}_{\mathrm{i} 5}, \mathrm{u}_{\mathrm{i}+15}, \mathrm{u}_{\mathrm{i}+13}\right): 2 \leq \mathrm{i} \leq \mathrm{n}-1\right]\right\}$

$f^{*}\left[P_{1}\right]=f\left(u_{i 1}\right)+f\left(u_{i 4}\right)+f\left(u_{i 1} u_{12}\right)+f\left(u_{i 2} u_{i 3}\right)+f\left(u_{i 3} u_{i 4}\right)$

$=\mathrm{i}+8 \mathrm{n}-2+2(\mathrm{i}-1)+2 \mathrm{n}+3-\mathrm{i}+5 \mathrm{n}-2+(\mathrm{n}+1-\mathrm{i})+6 \mathrm{n}-2+(\mathrm{n}+1-\mathrm{i})$

$=23 n-3$

$f^{*}\left[P_{2}\right]=f\left(u_{i 5}\right)+f\left(u_{i+1,3}\right)+f\left(u_{5} u_{i+1,3}\right)+f\left(u_{i+1,5} u_{i+1,3}\right)$

$=2 \mathrm{n}+2+\mathrm{i}-1+8 \mathrm{n}-1+2(\mathrm{i}-1)+5 \mathrm{n}-3-2(\mathrm{i}-2)+7 \mathrm{n}-2+\mathrm{n}-\mathrm{i}$

$=23 n-3$

$\mathrm{f}^{*}\left[\mathrm{P}_{3}\right]=\mathrm{f}\left(\mathrm{u}_{\mathrm{i} 3}\right)+\mathrm{f}\left(\mathrm{u}_{23}\right)+\mathrm{f}\left(\mathrm{u}_{\mathrm{i} 3} \mathrm{u}_{15}\right)+\mathrm{f}\left(\mathrm{u}_{15} \mathrm{u}_{25}\right)+\mathrm{f}\left(\mathrm{u}_{25} \mathrm{u}_{23}\right)$

$=\mathrm{n}+1+8 \mathrm{n}-1+2(\mathrm{i}-1)+5 \mathrm{n}-3-2(\mathrm{i}-2)+7 \mathrm{n}-2+\mathrm{n}-\mathrm{i}$

$=23 \mathrm{n}-3$

From (A), (B) and (C), we conclude that $\mathrm{G}$ admits $\psi$ - magic graphoidal total labeling. Hence, $\mathrm{T}_{(\mathrm{n})}$ is magic graphoidal. 
For example, consider the graph $\mathrm{T}_{(3)}$ shown in figure 4.

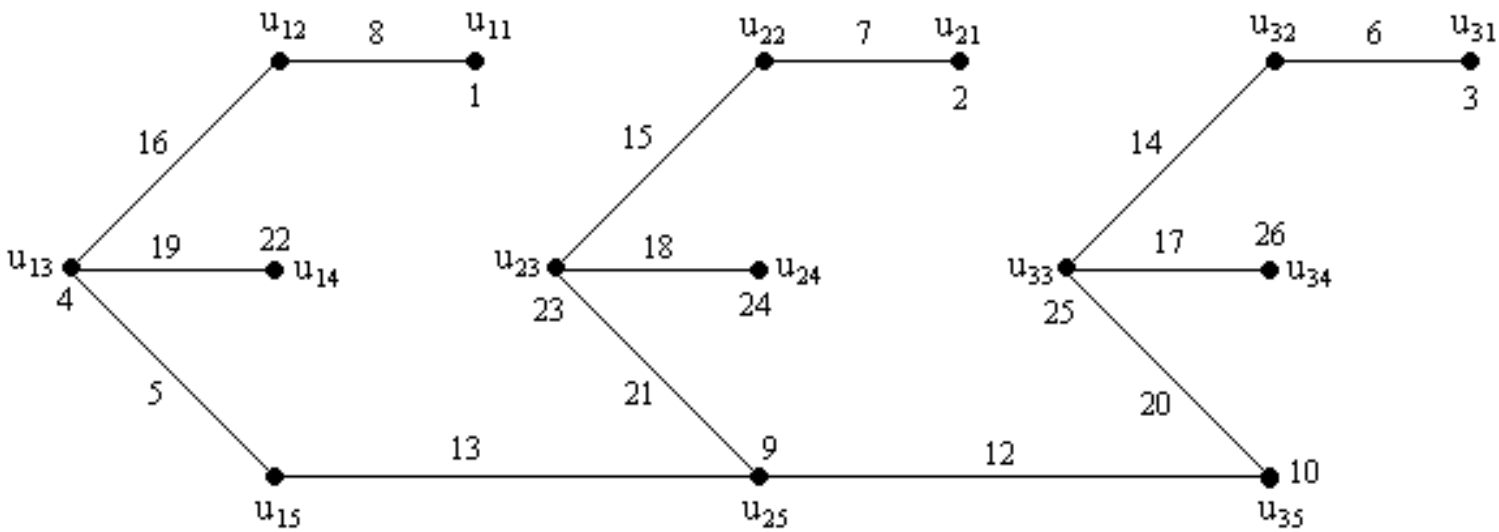

Figure $4 \mathrm{~T}_{(3)}$

Clearly, $\psi=\left\{\left(\mathrm{u}_{11}, \mathrm{u}_{12}, \mathrm{u}_{13}, \mathrm{u}_{14}\right),\left(\mathrm{u}_{21}, \mathrm{u}_{22}, \mathrm{u}_{23}, \mathrm{u}_{24}\right),\left(\mathrm{u}_{31}, \mathrm{u}_{32}, \mathrm{u}_{33}, \mathrm{u}_{34}\right),\left(\mathrm{u}_{13}, \mathrm{u}_{15}, \mathrm{u}_{25}, \mathrm{u}_{23}\right),\left(\mathrm{u}_{25}, \mathrm{u}_{35}, \mathrm{u}_{33}\right)\right\}$ is a minimum graphoidal cover and $\mathrm{T}_{(3)}$ is magic graphoidal. Here the constant $\mathrm{K}=66$

3.5. Theorem : The graph Double Crowned Star $K_{1, n} \odot K_{1}$ is magic graphoidal.

Proof : Let $\mathrm{G}=\mathrm{K}_{1, \mathrm{n}} \odot 2 \mathrm{~K}_{1}$

$\mathrm{V}(\mathrm{G})=\left\{\mathrm{u},\left[\mathrm{u}_{\mathrm{i}}: 1 \leq \mathrm{i} \leq \mathrm{n}\right],\left[\left(\mathrm{u}_{\mathrm{i} 1}, \mathrm{u}_{\mathrm{i} 2}\right): 1 \leq \mathrm{i} \leq \mathrm{n}\right]\right\}$ and

$\mathrm{E}(\mathrm{G})=\left\{\left[\left(\mathrm{uu}_{\mathrm{i}}\right): 1 \leq \mathrm{i} \leq \mathrm{n}\right] \cup\left[\left(\mathrm{u}_{\mathrm{i}} \mathrm{u}_{\mathrm{i} 1}\right) \cup\left(\mathrm{u}_{\mathrm{i}} \mathrm{u}_{\mathrm{i} 2}\right): 1 \leq \mathrm{i} \leq \mathrm{n}\right]\right\}$

Define $f: V \cup E \rightarrow\{1,2, \ldots, p+q\}$ by

\begin{tabular}{|c|c|c|}
\hline $\mathrm{f}(\mathrm{u})$ & $=2 n+3$ & \\
\hline $\mathrm{f}\left(\mathrm{u}_{\mathrm{i} 1}\right)$ & $=\mathrm{i}$ & $1 \leq \mathrm{i} \leq \mathrm{n}$ \\
\hline$f\left(u_{1}\right)$ & $=\mathrm{n}+1$ & \\
\hline$f\left(u_{2}\right)$ & $=\mathrm{n}+2$ & \\
\hline$f\left(u_{n+1-i, 2}\right)$ & $=n+2+i$ & $1 \leq \mathrm{i} \leq \mathrm{n}$ \\
\hline $\mathrm{f}\left(\mathrm{u}_{\mathrm{i}} \mathrm{u}_{\mathrm{i}, 1}\right)$ & $=2 n+3+i$ & $1 \leq \mathrm{i} \leq \mathrm{n}$ \\
\hline $\mathrm{f}\left(\mathrm{uu}_{1}\right)$ & $=3 n+4$ & \\
\hline $\mathrm{f}\left(\mathrm{u}_{2+\mathrm{i}}\right)$ & $=3 n+4+i$ & $1 \leq \mathrm{i} \leq \mathrm{n}-2$ \\
\hline$f\left(u_{n+1-i}\right)$ & $=4 n+2+i$ & $1 \leq \mathrm{i} \leq \mathrm{n}-1$ \\
\hline$f\left(u_{n+1-i} u_{n}\right.$ & $=5 n+1+i$ & $1 \leq \mathrm{i} \leq \mathrm{n}$ \\
\hline
\end{tabular}

Let $\psi=\left\{\mathrm{P}_{1}=\left(\mathrm{u}_{1}, \mathrm{u}, \mathrm{u}_{2}\right), \mathrm{P}_{2}=\left[\left(\mathrm{u}, \mathrm{u}_{\mathrm{i}}\right): 3 \leq \mathrm{i} \leq \mathrm{n}\right], \mathrm{P}_{3}=\left[\left(\mathrm{u}_{\mathrm{i} 1}, \mathrm{u}_{\mathrm{i}}, \mathrm{u}_{\mathrm{i} 2}\right): 1 \leq \mathrm{i} \leq \mathrm{n}\right]\right\}$

$$
\begin{aligned}
f^{*}\left[P_{1}\right] & =f\left(u_{1}\right)+f\left(u_{2}\right)+f\left(u_{1} u\right)+f\left(u_{2}\right) \\
& =n+1+n+2+3 n+4+5 n+1 \\
& =10 n+8
\end{aligned}
$$

For $3 \leq \mathrm{i} \leq \mathrm{n}$,

$$
\begin{aligned}
f^{*}\left[P_{2}\right] & =f(u)+f\left(u_{i}\right)+f\left(u_{i}\right) \\
& =2 n+3+3 n+4+i-2+4 n+2+n+1-i \\
& =10 n+8-
\end{aligned}
$$

For $1 \leq \mathrm{i} \leq \mathrm{n}$, 


$$
\begin{aligned}
f^{*}\left[P_{3}\right] & =f\left(u_{i 1}\right)+f\left(u_{i 2}\right)+f\left(u_{i 1} u_{i}\right)+f\left(u_{i} u_{i 2}\right) \\
& =i+n+2+n+1-i+2 n+3+i+5 n+1+n+1-i \\
& =10 n+8
\end{aligned}
$$

From (A), (B) and (C), we conclude that $\mathrm{G}$ admits $\psi$ - magic graphoidal total labeling. Hence, Double Crowned Star $\mathrm{K}_{1, \mathrm{n}} \odot 2 \mathrm{~K}_{1}$ is magic graphoidal.

For example, consider the graph $\mathrm{K}_{1,5} \odot 2 \mathrm{~K}_{1}$ shown in figure 5.

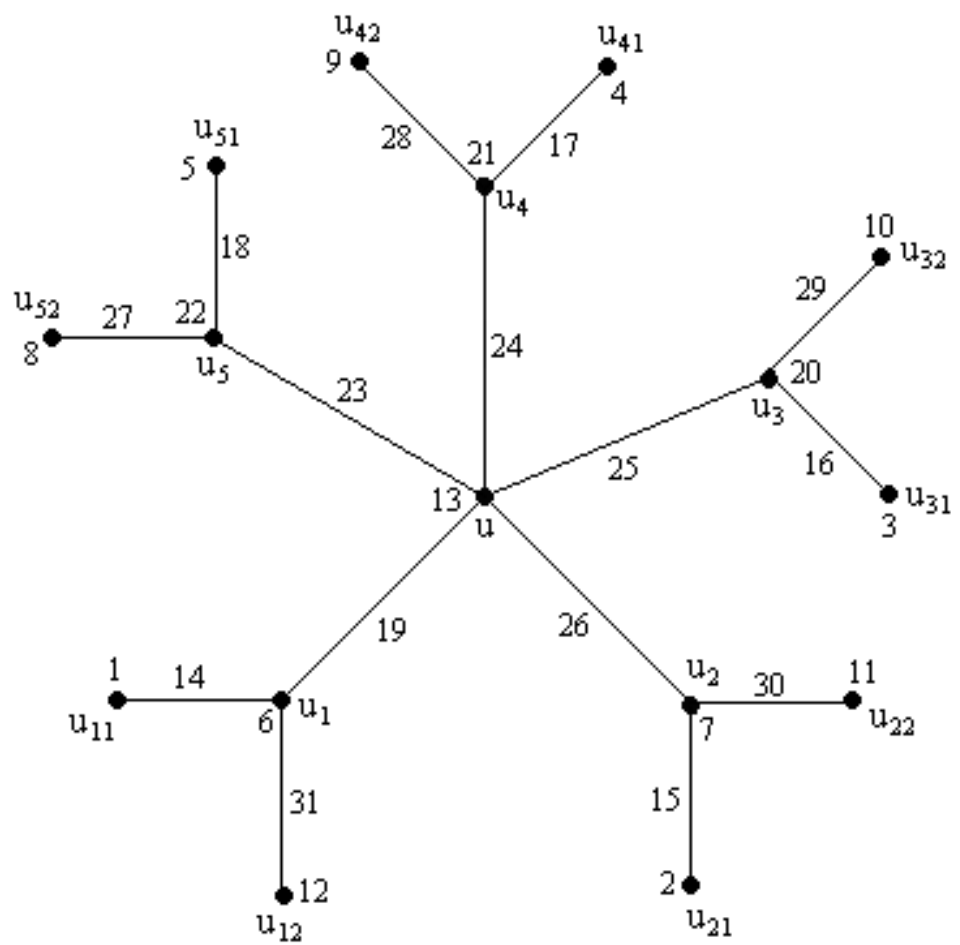

Figure 5. $\mathbf{K}_{\mathbf{1 , 5}} \odot \mathbf{2} \mathbf{K}_{1}$

Clearly, $\psi=\left\{\left(\mathrm{u}_{11}, \mathrm{u}_{1}, \mathrm{u}_{12}\right),\left(\mathrm{u}_{21}, \mathrm{u}_{2}, \mathrm{u}_{22}\right),\left(\mathrm{u}_{31}, \mathrm{u}_{3}, \mathrm{u}_{32}\right),\left(\mathrm{u}_{41}, \mathrm{u}_{4}, \mathrm{u}_{42}\right),\left(\mathrm{u}_{51}, \mathrm{u}_{5}, \mathrm{u}_{52}\right),\left(\mathrm{u}_{1}, \mathrm{u}, \mathrm{v}, \mathrm{u}_{2}\right),\left(\mathrm{u}, \mathrm{u}_{3}\right)\right.$, $\left.\left(\mathrm{u}, \mathrm{u}_{4}\right),\left(\mathrm{u}, \mathrm{u}_{5}\right)\right\}$ is a minimum graphoidal cover and $\mathrm{K}_{1,5} \odot 2 \mathrm{~K}_{1}$ is magic graphoidal. Here the constant $\mathrm{K}=58$.

3.6. Theorem : $\mathrm{P}_{\mathrm{m}} \bigodot_{2 \mathrm{~K}_{1}}$ is magical graphoidal.

Proof : Let $\mathrm{G}=\mathrm{P}_{\mathrm{m}} \circlearrowright 2 \mathrm{~K}_{1}$

$$
\begin{aligned}
& \mathrm{V}(\mathrm{G})=\left\{\left(\mathrm{u}_{\mathrm{i}}: 1 \leq \mathrm{i} \leq \mathrm{m}\right),\left(\mathrm{u}_{\mathrm{ij}}: 1 \leq \mathrm{i} \leq \mathrm{m}, 1 \leq \mathrm{j} \leq 2\right)\right\} \text { and } \\
& \mathrm{E}(\mathrm{G})=\left\{\left[\left(\mathrm{u}_{\mathrm{i}} \mathrm{u}_{\mathrm{i}+1}\right): 1 \leq \mathrm{i} \leq \mathrm{m}-1\right] \cup\left[\left(\mathrm{u}_{\mathrm{i}} \mathrm{u}_{\mathrm{ij}}\right): 1 \leq \mathrm{i} \leq \mathrm{m}, 1 \leq \mathrm{j} \leq 2\right]\right\}
\end{aligned}
$$

Let $\psi=\left\{\mathrm{P}_{1}=\left[\left(\mathrm{u}_{\mathrm{i} 1}, \mathrm{u}_{\mathrm{i}}, \mathrm{u}_{\mathrm{i} 2}\right): 1 \leq \mathrm{i} \leq \mathrm{m}\right], \mathrm{P}_{2}=\left[\left(\mathrm{u}_{\mathrm{i}}, \mathrm{u}_{\mathrm{i}+1}\right): 1 \leq \mathrm{i} \leq \mathrm{m}-1\right]\right\}$

Define $\mathrm{f}: \mathrm{V} \rightarrow\{0,1,2, \ldots, 6 \mathrm{~m}-1\}$ by

$$
\begin{aligned}
& \mathrm{f}\left(\mathrm{u}_{\mathrm{i}} \mathrm{u}_{\mathrm{i} 1}\right)=\mathrm{i} \quad 1 \leq \mathrm{i} \leq \mathrm{m} \\
& \mathrm{f}\left(\mathrm{u}_{\mathrm{i}} \mathrm{u}_{\mathrm{i} 2}\right)=2 \mathrm{~m}+1-\mathrm{i} \quad 1 \leq \mathrm{i} \leq \mathrm{m}
\end{aligned}
$$

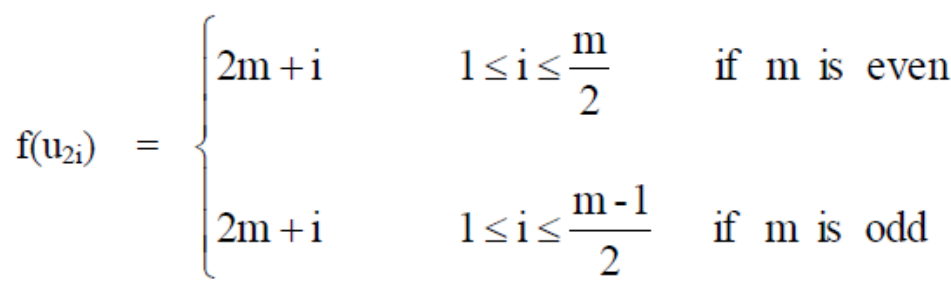




$$
\begin{aligned}
& \mathrm{f}\left(\mathrm{u}_{\mathrm{i} 1}\right)= \begin{cases}\frac{5 \mathrm{~m}}{2}+\mathrm{i} & 1 \leq \mathrm{i} \leq \mathrm{m} \text { if } \mathrm{m} \text { is even } \\
\frac{5 \mathrm{~m}-1}{2}+\mathrm{i} & 1 \leq \mathrm{i} \leq \mathrm{m} \text { if } \mathrm{m} \text { is odd }\end{cases}
\end{aligned}
$$

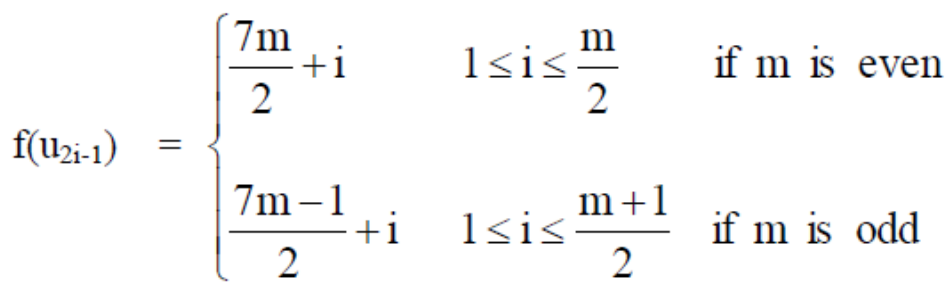

$$
\begin{aligned}
& \mathrm{f}\left(\mathrm{u}_{\mathrm{m}+1-\mathrm{i}} \mathrm{u}_{\mathrm{m}-\mathrm{i}}\right) \quad=4 \mathrm{~m}+\mathrm{i} \quad 1 \leq \mathrm{i} \leq \mathrm{m}-1 \\
& \mathrm{f}\left(\mathrm{u}_{\mathrm{m}+1-\mathrm{i}, 2)}\right)=5 \mathrm{~m}-1+\mathrm{i} \quad 1 \leq \mathrm{i} \leq \mathrm{m}
\end{aligned}
$$

Case (i) : when $\mathrm{m}$ is odd

For $1 \leq \mathrm{i} \leq \mathrm{m}$,

$$
\begin{aligned}
f^{*}\left[P_{1}\right] & =f\left(u_{i 1}\right)+f\left(u_{i 2}\right)+f\left(u_{i 1} u_{i}\right)+f\left(u_{i} u_{i 2}\right) \\
& =\frac{5 m-1}{2}+i+5 m-1+m+1-i+i+2 m+1-i \\
& =\frac{21 m+1}{2}-(A)
\end{aligned}
$$

For $1 \leq \mathrm{i} \leq \mathrm{m}-1, \mathrm{i} \equiv 1 \bmod 2$

$$
\begin{aligned}
f^{*}\left[P_{2}\right] & =f\left(u_{i}\right)+f\left(u_{i+1}\right)+f\left(u_{i} u_{i+1}\right) \\
& =\frac{7 m-1}{2}+\frac{i+1}{2}+2 m+\frac{i+1}{2}+4 m+m-i \\
& =\frac{21 m+1}{2}-(B)
\end{aligned}
$$

For $1 \leq \mathrm{i} \leq \mathrm{m}-1, \mathrm{i} \equiv 0 \bmod 2$

$$
\begin{aligned}
f^{*}\left[P_{2}\right]= & f\left(u_{i}\right)+f\left(u_{i+1}\right)+f\left(u_{i} u_{i+1}\right) \\
& =2 m+\frac{i}{2}+\frac{7 m-1}{2}+\frac{i+2}{2}+4 m+m-i \\
& =\frac{21 m+1}{2}--(C)
\end{aligned}
$$

From (A), (B) and (C), we conclude that $\mathrm{G}$ admits $\psi$ - magic graphoidal total labeling. Hence, $\mathrm{P}_{\mathrm{m}} \bigodot 2 \mathrm{~K}_{1}$ (m-odd) is magic graphoidal.

For example, consider the graph $\mathrm{P}_{5} \bigodot_{2 \mathrm{~K}_{1}}$ shown in figure 6.1 .

Case (ii) : when $m$ is even

For $1 \leq \mathrm{i} \leq \mathrm{m}$,

$f^{*}\left[P_{1}\right]=f\left(u_{i 1}\right)+f\left(u_{i 2}\right)+f\left(u_{i 1} u_{i}\right)+f\left(u_{i} u_{i 2}\right)$ 


$$
\begin{aligned}
& \frac{5 \mathrm{~m}}{2}=+\mathrm{i}+5 \mathrm{~m}-1+\mathrm{m}+1-\mathrm{i}+\mathrm{i}+2 \mathrm{~m}+1-\mathrm{i} \\
& \frac{21 \mathrm{~m}+2}{2} \text {----- (A) }
\end{aligned}
$$

For $1 \leq \mathrm{i} \leq \mathrm{m}-1, \mathrm{i} \equiv 1 \bmod 2$

$$
\begin{aligned}
f^{*}\left[P_{2}\right]=f\left(u_{i}\right) & +f\left(u_{i+1}\right)+f\left(u_{i} u_{i+1}\right) \\
= & \frac{7 m}{2}+\frac{i+1}{2}+2 m+\frac{i+1}{2}+4 m+m-i \\
= & \frac{21 \mathrm{~m}+2}{2}
\end{aligned}
$$

For $1 \leq \mathrm{i} \leq \mathrm{m}-1, \mathrm{i} \equiv 0 \bmod 2$

$$
\begin{aligned}
f^{*}\left[P_{2}\right]=f\left(u_{i}\right) & +f\left(u_{i+1}\right)+f\left(u_{i} u_{i+1}\right) \\
& =2 m+\frac{i}{2}+\frac{7 m}{2}+\frac{i+2}{2}+4 m+m-i \\
& =\frac{21 \mathrm{~m}+2}{2}
\end{aligned}
$$

From (A), (B) and (C), we conclude that $\mathrm{G}$ admits $\psi$ - magic graphoidal total labeling. Hence, $\mathrm{P}_{\mathrm{m}} \oslash 2 \mathrm{~K}_{1}$ (m-even) is magic graphoidal.

For example, consider the graph $\mathrm{P}_{4} \bigodot 2 \mathrm{~K}_{1}$ shown in figure 6.2.

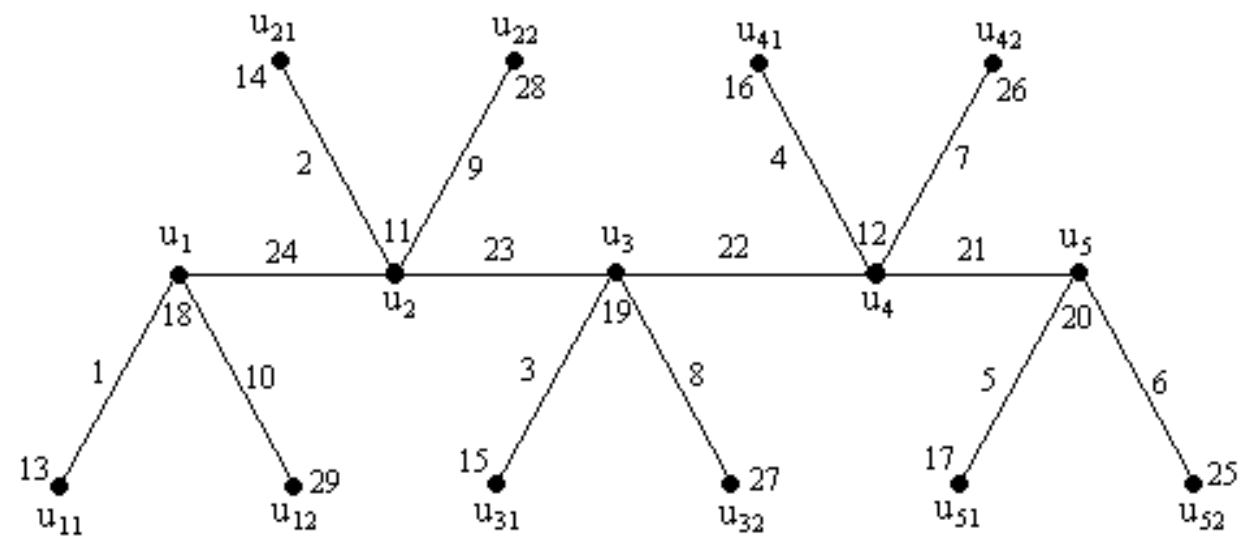

Figure 6.1 $P_{5} \bigodot 2 K_{1}$

Clearly, $\psi=\left\{\left(\mathrm{u}_{11}, \mathrm{u}_{1}, \mathrm{u}_{12}\right), \quad\left(\mathrm{u}_{21}, \mathrm{u}_{2}, \mathrm{u}_{22}\right), \quad\left(\mathrm{u}_{31}, \mathrm{u}_{3}, \mathrm{u}_{32}\right), \quad\left(\mathrm{u}_{41}, \mathrm{u}_{4}, \mathrm{u}_{42}\right), \quad\left(\mathrm{u}_{51}, \mathrm{u}_{5}, \mathrm{u}_{52}\right), \quad\left(\mathrm{u}_{1}, \mathrm{u}_{2}\right), \quad\left(\mathrm{u}_{2}, \mathrm{u}_{3}\right)\right.$, $\left.\left(\mathrm{u}_{3}, \mathrm{u}_{4}\right),\left(\mathrm{u}_{4}, \mathrm{u}_{5}\right)\right\}$ is a minimum graphoidal cover and $\mathrm{P}_{4} \circlearrowright 2 \mathrm{~K}_{1}$ is magic graphoidal. Here the constant $\mathrm{K}=53$. 


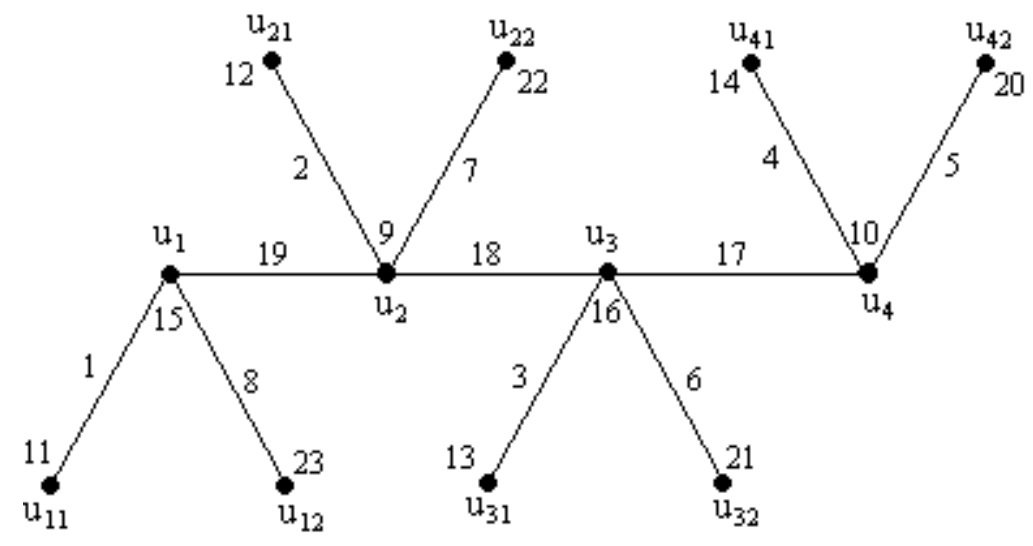

Figure 6.2 $\mathbf{P}_{4} \circlearrowleft 2 K_{1}$

Clearly, $\psi=\left\{\left(\mathrm{u}_{11}, \mathrm{u}_{1}, \mathrm{u}_{12}\right),\left(\mathrm{u}_{21}, \mathrm{u}_{2}, \mathrm{u}_{22}\right),\left(\mathrm{u}_{31}, \mathrm{u}_{3}, \mathrm{u}_{32}\right),\left(\mathrm{u}_{41}, \mathrm{u}_{4}, \mathrm{u}_{42}\right),\left(\mathrm{u}_{1}, \mathrm{u}_{2}\right),\left(\mathrm{u}_{2}, \mathrm{u}_{3}\right),\left(\mathrm{u}_{3}, \mathrm{u}_{4}\right)\right\}$ is a minimum graphoidal cover and $\mathrm{P}_{4} \bigodot_{2 \mathrm{~K}_{1}}$ is magic graphoidal. Here the constant $\mathrm{K}=43$.

3.7. Theorem : $\mathrm{P}_{\mathrm{m}} \bigodot_{\mathrm{K}_{1,3}}$ is magic graphoidal.

Proof : Let $\mathrm{G}=\mathrm{P}_{\mathrm{m}} \precsim \mathrm{K}_{1,3}$

$\mathrm{V}(\mathrm{G})=\left\{\left[\left(\mathrm{u}_{\mathrm{i}} \mathrm{v}_{\mathrm{i}}\right): 1 \leq \mathrm{i} \leq \mathrm{m}\right],\left[\left(\mathrm{v}_{\mathrm{ij}}\right): 1 \leq \mathrm{i} \leq \mathrm{m}, 1 \leq \mathrm{i} \leq 3\right]\right\}$

$\mathrm{E}(\mathrm{G})=\left\{\left[\left(\mathrm{u}_{\mathrm{i}} \mathrm{u}_{\mathrm{i}+1}\right): 1 \leq \mathrm{i} \leq \mathrm{m}-1\right] \cup\left[\left(\mathrm{v}_{\mathrm{i}} \mathrm{v}_{\mathrm{ij}}\right): 1 \leq \mathrm{i} \leq \mathrm{m}, 1 \leq \mathrm{j} \leq 3\right]\right\}$ with $\mathrm{u}_{\mathrm{i}}=\mathrm{u}_{\mathrm{i} 3}, 1 \leq \mathrm{i} \leq \mathrm{m}$

Let $\psi=\left\{\mathrm{P}_{1}=\left[\left(\mathrm{v}_{\mathrm{i} 1}, \mathrm{v}_{\mathrm{i}}, \mathrm{v}_{\mathrm{i} 2}\right): 1 \leq \mathrm{i} \leq \mathrm{m}\right], \mathrm{P}_{2}=\left[\left(\mathrm{v}_{\mathrm{i}}, \mathrm{u}_{\mathrm{i}}, \mathrm{u}_{\mathrm{i}+1}\right): 1 \leq \mathrm{i} \leq \mathrm{m}-2\right]\right.$,

$$
\left.\mathrm{P}_{3}=\left(\mathrm{v}_{\mathrm{m}-1}, \mathrm{u}_{\mathrm{m}-1}, \mathrm{u}_{\mathrm{m}}, \mathrm{v}_{\mathrm{m}}\right)\right\}
$$

Define $\mathrm{f}: \mathrm{V} \cup \mathrm{E} \rightarrow\{1,2, \ldots, 8 \mathrm{~m}-1\}$ by

\begin{tabular}{|c|c|c|}
\hline$f\left(v_{m}\right)$ & $=1$ & \\
\hline $\mathrm{f}\left(\mathrm{v}_{\mathrm{i}} \mathrm{v}_{\mathrm{i} 1}\right)$ & $=\mathrm{i}+1$ & $1 \leq \mathrm{i} \leq \mathrm{m}$ \\
\hline $\mathrm{f}\left(\mathrm{u}_{\mathrm{i}} \mathrm{v}_{\mathrm{i}}\right)$ & $=\mathrm{m}+1+\mathrm{i}$ & $1 \leq \mathrm{i} \leq \mathrm{m}-1$ \\
\hline$f\left(u_{m+1-i} u_{m-i}\right)$ & $=2 \mathrm{~m}+\mathrm{i}$ & $1 \leq \mathrm{i} \leq \mathrm{m}-1$ \\
\hline$f\left(v_{m+1-i} V_{m+1-i, 2}\right)$ & $=3 \mathrm{~m}-1+\mathrm{i}$ & $1 \leq \mathrm{i} \leq \mathrm{m}$ \\
\hline $\mathrm{f}\left(\mathrm{v}_{\mathrm{i} 1}\right)$ & $=4 m-1+i$ & $1 \leq \mathrm{i} \leq \mathrm{m}$ \\
\hline$f\left(v_{i}\right)$ & $=5 \mathrm{~m}-1+\mathrm{i}$ & $1 \leq \mathrm{i} \leq \mathrm{m}-1$ \\
\hline$f\left(u_{m} v_{m}\right)$ & $=6 \mathrm{~m}-1$ & \\
\hline $\mathrm{f}\left(\mathrm{u}_{\mathrm{m}-\mathrm{i}}\right)$ & $=6 \mathrm{~m}+\mathrm{i}$ & $1 \leq \mathrm{i} \leq \mathrm{m}-2$ \\
\hline$f\left(v_{m+1-i, 2}\right)$ & $=7 \mathrm{~m}-2+\mathrm{i}$ & $1 \leq \mathrm{i} \leq \mathrm{m}$ \\
\hline
\end{tabular}

For $1 \leq \mathrm{i} \leq \mathrm{m}$,

$$
\begin{aligned}
f^{*}\left[P_{1}\right]= & f\left(v_{i 1}\right)+f\left(v_{i 2}\right)+f\left(v_{i 1} v_{i}\right)+f\left(v_{i} v_{i 2}\right) \\
= & 4 m-1+i+7 m-2+m+1-i+i+1+3 m-1+m+1-i \\
= & 16 m-1--
\end{aligned}
$$

For $1 \leq \mathrm{i} \leq \mathrm{m}-2$,

$$
\begin{aligned}
f^{*}\left[P_{2}\right]= & f\left(v_{i}\right)+f\left(u_{i+1}\right)+f\left(v_{i} u_{i}\right)+f\left(u_{i} u_{i+1}\right) \\
& =5 m-1+i+6 m+(m-i-1)+m+1+i+2 m+m-i \\
& =16 m-1
\end{aligned}
$$




$$
\begin{aligned}
f^{*}\left[P_{3}\right]= & f\left(v_{m-1}\right)+f\left(v_{m}\right)+f\left(v_{m-1} u_{m-1}\right)+f\left(u_{m-1} u_{m}\right)+f\left(u_{m} v_{m}\right) \\
& =6 m-2+1+2 m+2 m+1+6 m-1 \\
& =16 m-1----(C)
\end{aligned}
$$

From (A), (B) and (C), we conclude that $\mathrm{G}$ admits $\psi$ - magic graphoidal total labeling. Hence, $\mathrm{P}_{\mathrm{m}}$ $\smile \mathrm{K}_{1,3}$ is magic graphoidal.

For example, consider the graph $\mathrm{P}_{4} \circlearrowright \mathrm{K}_{1,3}$ shown in figure 7 .

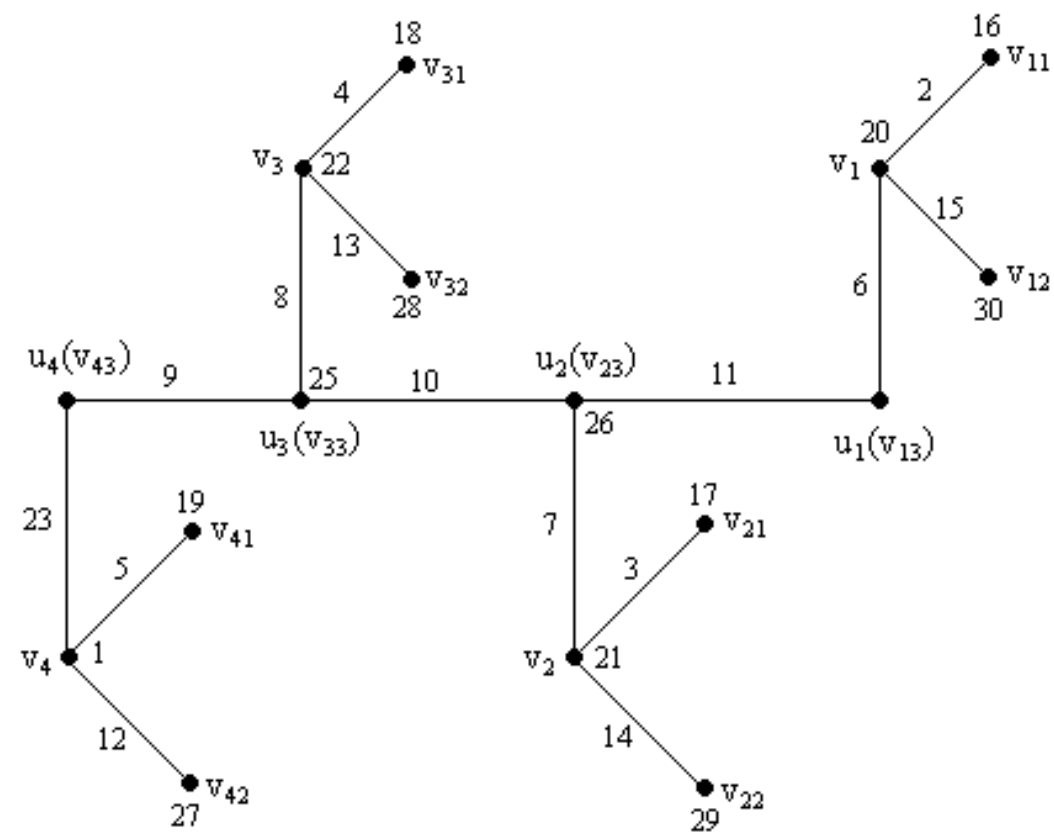

Figure 7. $\mathbf{P}_{\mathbf{4}} \bigodot \mathbf{K}_{1,3}$

Clearly, $\psi=\left\{\left(\mathrm{v}_{11}, \mathrm{v}_{1}, \mathrm{v}_{12}\right),\left(\mathrm{v}_{21}, \mathrm{v}_{2}, \mathrm{v}_{22}\right),\left(\mathrm{v}_{31}, \mathrm{v}_{3}, \mathrm{v}_{32}\right),\left(\mathrm{v}_{41}, \mathrm{v}_{4}, \mathrm{v}_{42}\right),\left(\mathrm{v}_{1}, \mathrm{u}_{1}, \mathrm{u}_{2}\right),\left(\mathrm{v}_{1}, \mathrm{u}_{2}, \mathrm{u}_{3}\right),\left(\mathrm{v}_{3}, \mathrm{u}_{3}, \mathrm{u}_{4}, \mathrm{v}_{4}\right)\right\}$ is magic graphoidal. Here the constant $\mathrm{K}=63$.

\section{REFERENCES}

[1] B.D.Acarya and E. Sampath Kumar, Graphoidal covers and Graphoidal covering number of a Graph, Indian J.Pure Appl. Math. 18(10).(1987).882-890.

[2] J.A. Gallian, A Dynamic Survey of graph labeling, The Electronic journal of Coimbinotorics 6 (2001) \# DS6.

[3] F. Harary, Graph Theory, Addition - Wesley publishing company Inc, USA, 1969.

[4] A. Nellai Murugan, Magic graphoidal on $S_{m}, n$ Outreach, A Multidisciplinary Refreed Journal , Vol. 4 (2011-12), 75-78.

[5] A. Nellai Murugan and A. Nagarajan, Magic Graphoidal on Special type of Unicyclic Graphs, Scientia Magna, Vol.7, (2011), No. 1, 99-105.

[6] A. Nellai Murugan and A.Nagarajan Magic graphidal of path related graphs Advance Journal of Physical sciences, Access International Journals, Vol 1(2) pp 14-25, December 2012

[7] A. Nellai Murugan, Magic graphiadal on join of two graphs, International Journal of mathematical combinatorics. Vol 4(2012), 103-115

[8] A. Nellai Murugan, Magic graphiadal on product graphs, International journal of innovative research \& development Vol 1(8),2012, 44-53

[9] C. Packiam and S. Arumugam, On the Graphoidal covering number of a Graph. Indian J.Pure Appl. Math. 20 (1989), 330-333. 